\title{
PHOTOMETRIC AND SPECTROSCOPIC VARIATIONS OF THE SYMBIOTIC STAR
} EG ANDROME DAE

\author{
A. Skopal ${ }^{1}$, D. Chochol ${ }^{1}$, A. Vittone ${ }^{2}$, A. Mammano ${ }^{3}$ \\ 1 Astronomical Institute, Slovak Academy of Sciences, \\ 05960 Tatranská Iomnica, Czechoslovakia \\ 2 Osservatorio Astronomico Capodimonte, \\ Via Moiariello 16, 80131 Napol1, Italy \\ 3 Universita di Messina,Via C. Battisti 90,Messina, Italy
}

EG And 1s a symbiotic binary system. The cool component is an M3 III star, the hot one is a subdwarf with temperature $60250 \mathrm{~K}$ and luminom sity 1.45 Io (Boyarchuk, 1985). The eclipsing nature of the system suggested from UV spectroscopy by Oliversen et al. (1985) was confirmed photometrically by Chochol et al. (1987). The c1rcular spectroscopic orbit of cool component determined by oliversen et al. (1985) supposing the orbital period 470 days leads to $f(m)=3.2 \times 10^{-2} \mathrm{M}_{\odot}$ and detachedconfiguration.

The UBV photometry of EG And was obtained in 1985-87 by $0.6 \mathrm{~m}$ telescope at Skalnaté Pleso Observatory. The differential photometry $\Delta m=m(E G$ And $)-m(H D$ 3914) in U colour is presented in Fig. 1. Our observations cover two recent minima, which occured in 1985 and 1986-87. The eclipse is most pronounced in U colour. The details about UBV photoelectric observations of EG And are in the paper of Chochol and skopal (1987), where orbital period 480 days was suggested. The phase in Fig. 1 was computed using the period 481.7 days, which we obtained from spectroscopic observations. Therefore a new ephemeris for minima is:

$$
J D_{\min }=2446336.7+481.7 \text { days } \times \mathrm{E} \text {. }
$$

As it 1s possible to see on F1g. 1, the descending branch of the minimum is steeper than the ascending one, that could be caused by an elliptical orbit. This conclusion has been confirmed from spectroscopic elements.

The spectroscopic observations of EG And have been carried out at Asiago Astrophysical Observatory in 1966-69, 1972, 1978, 1983. Altogether 20 spectra were obtained by $1.22 \mathrm{~m}$ telescope with a prism spectrograph with dispersion of $6 \mathrm{~nm} / \mathrm{mm}$ at $\mathrm{H}_{\mathrm{y}}$. Two spectra with a dispersion $1.8 \mathrm{~nm} / \mathrm{mm}$ were obtained by $2 \mathrm{~m}$ telescope at ondrejov observatory in 1976, two spectra with the same dispersion were obtained by $0.9 \mathrm{~m}$ telescope at Torun Observatory in 1986. We measured radial velocities of neutral metals to obtain information about the motion of cool component. The data are presented in Fig. 2, where also the 


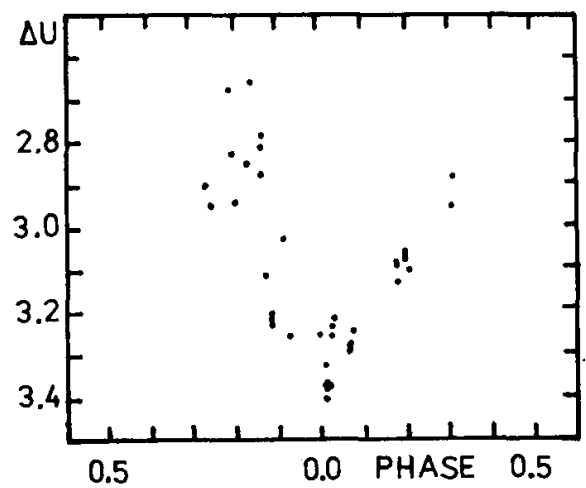

Figure 1. Differential U photometry of EG And.

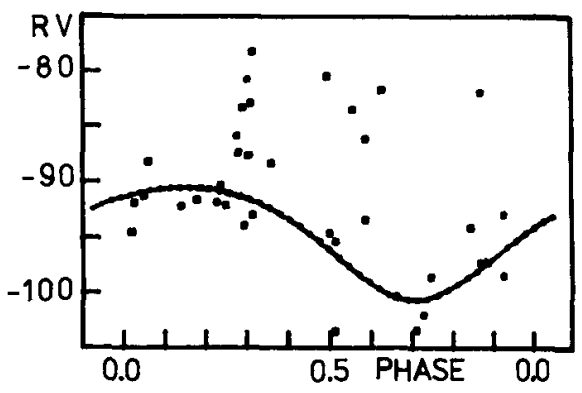

Pigure 2. Phase diagram of the radial velocities of neutral metals and spectroscopic orbit of EG And.

radial velocities of cool component published by Oliversen et al. (1985) and Garcia (1986) are included. At first we computed the phases of radial velocities with the period 480 days. We obtained a phase distribution of radial velocities close to this one on Fig. 2. It was obvious that radial velocities exceeding $-87 \mathrm{~km} / \mathrm{s}$ can not reflect the orbital motion, so we removed them. Orbital elements were computed using the code SPEI (Horm, 1987):

$$
\begin{array}{rlrl}
P & =(481.7 \pm 2.1) \text { days } & & T(\text { periastr. })=2442324 \mathrm{JD} \\
e & =0.17 \pm 0.12 & & T(\min .)=2442432 \mathrm{JD} \\
\omega & =169^{\circ} \pm 43^{\circ} & & \mathrm{f}(\mathrm{m})=6.18 \times 10^{-3} \mathrm{H}_{\Theta} \\
\hat{T}=-94 . \overline{7} \pm 0.4 \mathrm{~km} / \mathrm{s} & \mathrm{a}_{1} \mathrm{sin} 1=47.4 \mathrm{R}_{\odot}
\end{array}
$$

Possible masses of components are e.g. $0.5 \mathrm{M}_{0}$ for a hot component and $4 \mathrm{M}_{0}$ for a cool one $\left(1=90^{\circ}\right)$. The phases in Fig.2 were computed according to the ephemeris (1). All excluded radial velocities (except the $\mathrm{RV}=-81.6$ in the phase 0.86 , published by Garcia (1986)) were measured by us and occurred in the phases 0.29-0.63, when the cool component was behind the hot one. A nebulosity around the hot component can Influence the absorption lines of neutral metals. It is interesting to note that the strong emission lines of hydrogen were present in all excluded spectra.

The mass function determined by us is much smaller than the value determined by Oliversen et al. (1985), which leads to detached configuration. If our elements are real, the cool component easily overflows the Roche lobe.

REFERENCES

Boyarchuk, A.A.: 1985, In: Proc. ESA Workshop: Recent Results on Cataclyamic Variables, ESA SP - 236, 97.

Chochol, D., Skopal, A.: 1987, Astrofiz. Issled. BAN (in press). Chochol, D., Skopal, A., Vittone, A., Mammano, A.: 1987, Astrophys. Space Sc1. 131, 755 .

Garc1a, M.R.: 1986, Astron. J. 21, 1400. Horn, J.: 1987, private communication. Oliversen, N.A., et al.: 1985, Astrophys. J. 295, 620. 